\title{
The Least Action Solving Method of MPPT for Micro-Inverter in Distributed PV Grid
}

\author{
Xiaoju Yin ${ }^{1,2}$, Fengge Zhang ${ }^{1}$, Yonggang Jiao ${ }^{1}$ and Zhenhe $\mathrm{Ju}^{2}$ \\ 1. School of Electrical Engineering, Shenyang University of Technology, \\ Shenyang, 110870, China \\ 2. School of Renewable Energy, Shenyang Institute of Engineering, Shenyang, \\ 110136, China \\ *Corresponding author: Insolyxj@163.com
}

\begin{abstract}
The solar radiation conditions and environmental factors on the shadow of the power generation resulted in loss of efficiency. In this paper, a simple structure with interleaved isolation converter according to the characteristics of the hardware circuit is proposed in order to improve the conversion efficiency of photovoltaic grid system. First, the hot spot effect and discretization of solar panels according to the characteristics of the microinverter with a lot of promotion is analyzed. The least action recursive method is involved, which has high computing precision, and therefore is more suitable for distributed microinverter system. The PC56F8245 chip is chosen to overcome the loss of photovoltaic energy and improve the conversion efficiency. The stability and high accuracy has been proved through the related experiments.
\end{abstract}

Keywords: PV grid, the least action, modeling, photovoltaic panels, inverter

\section{Introduction}

At present, photovoltaic generation system are not widely used because of low efficiency and high cost. Maximum power point tracking technology could make full use of photovoltaic cells to convert energy and further improve working efficiency of the solar cells, which should be an important link of PV grid connected generation. The commonly methods can be divided into three categories. First, perturbation incremental method; second, intelligent incremental method; third, differential method. Perturbation incremental method [1] is gradually closing to the maximum power through continuous sampling. The advantage of this method lies on the simple algorithm, however, the selection of initial value and step have a great influence on tracking accuracy and tracking speed, thus, it can not adapt to drastic changing of sunshine per day. And it has a possibility of occurring oscillation near maximum power point which will cause power loss. Sometimes a program control disorder may occur in the proceeding, which will cause erroneous judgment. Intelligent incremental methods including fuzzy control [2] and neural network control [3]. Fuzzy control establishes fuzzy rules between sunshine changing and conduction rate, improving the influence of sunshine at the same time.

The advantage of fuzzy control is that maximum power point can also be found when sunshine changes dramatically every day, which also causes oscillation through continuous sampling. Neural network control need to extract a large number of samples but cannot reach the actual application process. Differential method[4] is more commonly used nowadays. When opening or closing the derivative of the power and voltage by calculating, the relationship of all pipe parameters adjust to the size of conduction rate. It will reach the maximum power when the derivative of the power and voltage is zero. The advantages of this method are the less volatile near the maximum power point, so it can 
have a higher precision, but this method is complex, it needs a lot of parameters to be made sure parameter values are also difficult to determine.

The recursive algorithm is proposed to use the duty cycle of the voltage and current changes combined with the principle of least action and calculation process, where the minimum amount is determined by the action of the maximum power point. This method avoids many fuzzy calculation processes and improves the accuracy of the calculation.

\section{The Principle of Photovoltaic Grid Power Tracking System}

The solar cell voltage characteristic curve is shown in Figure1. We need to find the best current and voltage at the time of maximum power to improve the efficiency of the system.

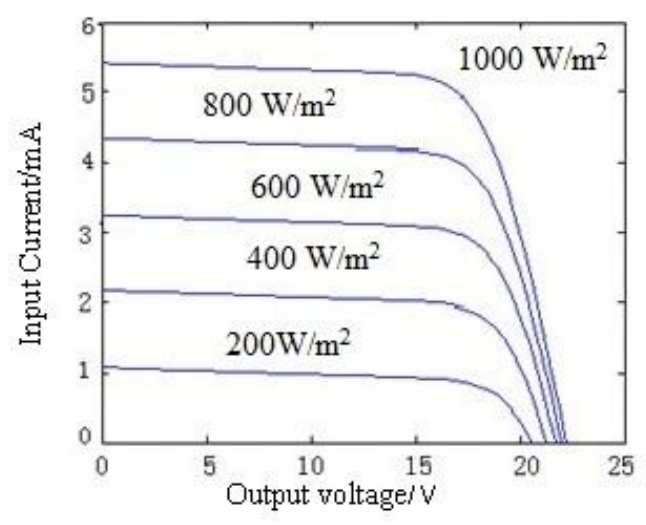

Figure 1. The volt-ampere characteristic curve of the solar cell

The PV grid system generally consists of the MPPT process and the inverter process as shown in Figure 2. The system is consisted of solar panels, boost DC/DC, single voltage type PWM inverter and the AC Power Group. Controlling the output DC power DC/DC, inverter output is connected to the capacitor Cs and the inductor Lc of the panel, switch Tc (IGBT) output termination diode VDC and filter capacitor Cd, Cs is to ensure that the input current waveform is stable. Controller 1 controls the DC/DC power to achieve MPPT process. Controller 2 realizes the inverter output AC current control and inverter DC input voltage control, and no isolation.

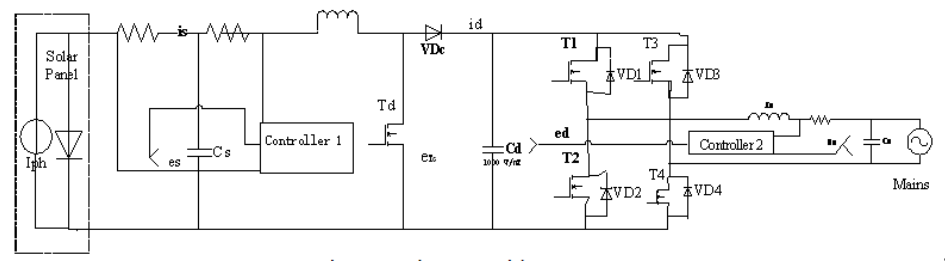

Figure 2. The PV Grid System

We can find the maximum power point and then carry out the string of components input to the inverter using separate MPPT device, which can control the strings of components independently as shown in Figure 3. The advantages of separate MPPT technique are in the case of the whole solar photovoltaic system and the costs do not change, the MPPT circuit originally installed in the inverter is decomposed into the front of each solar module string. The discrete of solar module parameters or the solar radiation conditions causes solar modules in parallel can help to overcome the energy loss under. 
The integration system can increase the generating capacity than conventional inverters and MPPT technology.

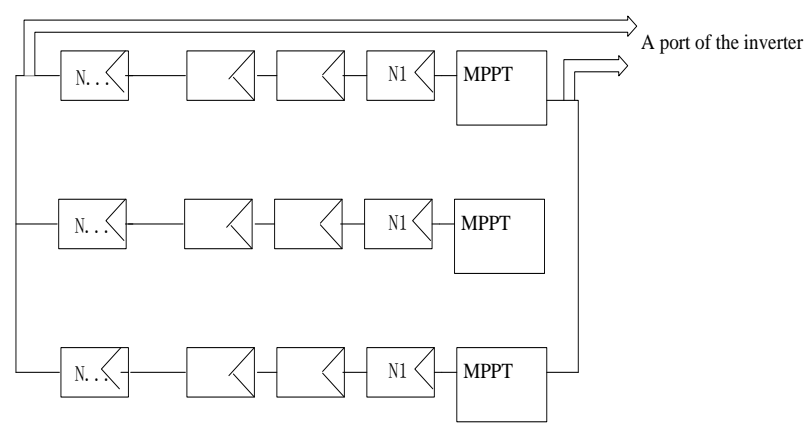

Figure 3. The Schematic of Distributed MPPT Technology

The schematic of control theory is shown in Figure4

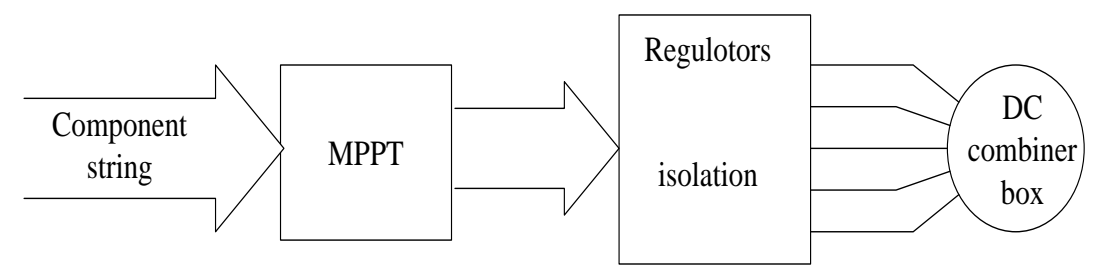

Figure 4. The Unit of Distributed MPPT

\section{Recursive Solution Method of Least Action for Maximum Power Point Tracking}

\subsection{The Judgment of Maximum Power Point Volt-Ampere Conditions}

The characteristics of solar cell can be expressed as:

$$
i_{s}=I_{p h}-I_{0}\left(e^{\frac{q}{K T} e_{s}}-1\right)
$$

where $i_{s}$ is the current of the solar cell, $e_{s}$ is the panel voltage, $I_{p h}$ is the short-circuit current of the solar cell, $I_{0}$ is the reverse saturation current of the diode, $q$ is the electron charge, $K$ is the Boltzmann constant, $T$ is the absolute temperature.

Denote $\phi=\frac{q}{K}$, and we can derive:

$$
i_{s}=I_{p h}-I_{0}\left(e^{\frac{\varphi}{T} e_{s}}-1\right)
$$

If $p_{s}$ is the power of panels, which is equal to the product of current and voltage, therefore:

$$
p_{s}=i_{s} e_{s}=\left[I_{p h}-I_{0}\left(e^{\frac{\phi}{T} e_{s}}-1\right)\right] e_{s}
$$

Based on the principle of least action, it can be drawn:

$$
\frac{d p_{s}}{d e_{s}}=i_{s}-\frac{e_{s} I_{0} \varphi}{T} e^{\frac{\varphi}{T} e_{s}}
$$

When the formula is equal to 0 , the point is the maximum power point, then: 


$$
i_{s}=\frac{e_{s} I_{0} \varphi}{T} e^{\frac{\varphi}{T} e_{s}}
$$

Therefore, as long as we confirm the judge of these holds, the maximum power point can be derived.

\subsection{Recurrence Relations}

IGBT is considered as an ideal switch according to Figure2, on and off state of the two present in each cycle. When Tc is on, it can be drawn:

$$
\begin{aligned}
& i_{s}=i_{L c}+C_{s} \frac{d}{d t} e_{s} \\
& e_{s}=L_{c} \frac{d}{d t} i_{L c}
\end{aligned}
$$

When tube Tc is off, that can be drawn:

$$
\begin{aligned}
& i_{s}=i_{L c}+C_{s} \frac{d}{d t} e_{s} \\
& e_{s}=L_{c} \frac{d}{d t} i_{L c}+e_{d}
\end{aligned}
$$

where, $i_{L c}$ is the current flowing through $L_{c}, e_{d}$ is the output voltage of DC/DC. Denoting conduction rate $\alpha$, the equation of state is:

$$
\left\{\begin{array}{l}
i_{s}=i_{L c}+C_{s} \frac{d}{d t} e_{s} \\
e_{s}=L_{c} \frac{d}{d t} i_{L c}+(1-\alpha) e_{d}
\end{array}\right.
$$

Denoting $e_{T c}=(1-\alpha) e_{d}$, the above equation can be arranged:

$$
\left\{\begin{array}{l}
\frac{d}{d t} e_{s}=-\frac{1}{C_{s}} i_{L c}+\frac{1}{C_{s}} i_{s} \\
\frac{d}{d t} i_{L c}=\frac{1}{L_{c}} e_{s}-\frac{1}{L_{c}} e_{T c}
\end{array}\right.
$$

Substituting the Eq. (1) into the equation (8), then:

$$
\left\{\begin{array}{l}
\frac{d}{d t} e_{s}=-\frac{1}{C_{s}} i_{L c}+\frac{1}{C_{s}}\left(I_{p h}+I_{0}-I_{0} e^{\frac{\phi}{T} e_{s}}\right) \\
\frac{d}{d t} i_{L c}=\frac{1}{L_{c}} e_{s}-\frac{1}{L_{c}} e_{T c}
\end{array}\right.
$$

Let $\Delta t$ take very small value, then:

$$
\left\{\begin{array}{l}
\frac{e_{s}(1)-e_{s}(0)}{\Delta t}=-\frac{1}{C_{s}} i_{L c}(0)+\frac{1}{C_{s}}\left(I_{p h}+I_{0}-I_{0} e^{\frac{\phi}{T} e_{s}(0)}\right) \\
\frac{i_{L c}(1)-i_{L c}(0)}{\Delta t}=\frac{1}{L_{c}} e_{s}(0)-\frac{1}{L_{c}} e_{T c}(0)
\end{array}\right.
$$




$$
\left\{\begin{array}{l}
e_{s}(1)=\left(-\frac{1}{C_{s}} i_{L c}(0)+\frac{1}{C_{s}}\left(I_{p h}+I_{0}\right)-\frac{I_{0}}{C_{s}} e^{\frac{\phi}{T} e_{s}(0)}\right) \Delta t+e_{s}(0) \\
i_{L c}(1)=\left(\frac{1}{L_{c}} e_{s}(0)-\frac{1}{L_{c}} e_{T c}(0)\right) \Delta t+i_{L c}(0)
\end{array}\right.
$$

\subsection{Recursive Algorithm for Computing Model}

According to the judgment conditions in subsections 3.1 and 3.2 and recurrence relations, the recursive algorithm implementation process are shown in Figure 3 , the calculation procedure can be illustrated as follows:

(1) Initial duty cycle is given, taking $\Delta t$ is equal to a very small value, detect the initial value $e_{s}(0), i_{L c}(0), e_{d}(0), T$.

(2) Calculate the initial value $e_{T c}(0)=(1-\alpha) e_{d}(0)$.

(3) If the value of $i_{s}(0)-e_{s}(0) I_{0} \frac{q}{K T} e^{\frac{q}{K T} e_{s}(0)}$ is equal to 0 , the procedure is ended; If not equal to 0 , then turns to the next step.

(4) Eq. (11) can be derived into following recurrence relations:

$$
\left\{\begin{array}{l}
e_{s}(k+1)=\left(-\frac{1}{C_{s}} i_{L c}(k)+\frac{1}{C_{s}}\left(I_{p h}+I_{0}\right)-\frac{I_{0}}{C_{s}} e^{\frac{\phi}{T} e_{s}(k)}\right) \Delta t+e_{s}(k) \\
i_{L c}(k+1)=\left(\frac{1}{L_{c}} e_{s}(k)-\frac{1}{L_{c}} e_{T c}(k)\right) \Delta t+i_{L c}(k)
\end{array}\right.
$$

(5) According to the obtained values from $e_{s}(k+1)$, substituting it into the equation $i_{s}(k+1)=I_{p h}-I_{0}\left(e^{\frac{q}{K T} e_{s}(k+1)}-1\right)$, we can calculate the values of $i_{s}(k+1)$

(6) If $i_{s}(k+1)-e_{s}(k+1) I_{0} \frac{q}{K T} e^{\frac{q}{K T} e_{s}(k+1)}$ is equal to 0 , the procedure is ended; if not, we change the value of $e_{T c}(k)$, if $e_{T c}(k+1)=e_{T c}(k) \pm \Delta e_{T c}(k)$, according to Eq. (8), continue to judge until the value is 0 . This moment, calculate the value of $e_{s}(k+1)$ as the voltage of the maximum power point while the value of $i_{s}(k+1)$ is the current of the maximum power point.

(7) According to the definition $e_{T c}=(1-\alpha) e_{d}$, the duty cycle is equal to:

$$
\alpha=1-\frac{e_{T c}}{e_{d}}
$$

Finally, we need assign the value of the duty cycle to the system.

\subsection{Determination of Parameter Values}

The two parameters $I_{0}, \varphi$ does not change with sunshine and temperature, when the solar panels and power systems is fixed.

Then, the formula is related to the following four changing values, the short-circuit 
current $I_{s c}$, the temperature $T$, the voltage value $e$, the current $i$, then we can get:

$$
i=I_{s c}-I_{0}\left(e^{\frac{\varphi}{T} e}-1\right)
$$

Then we can conclude:

$$
I_{s c}=i-I_{0}\left(e^{\frac{\varphi}{T} e}-1\right)
$$

Thus, the voltage value $e$, the current value $i$, we can calculate the short-circuit current $I_{s c}$ by sampling the current temperature $T$.

\subsection{A/D Sampling Parameters}

The initial sampling of maximum power point tracking including: the temperature $T$, the voltage value of the panel $e$, the panel current $i$, the inductor current $i_{L c}$, and the output voltage value $e_{d}$.

\section{System Implementation}

\subsection{Hardware Implementation}

MPPT experiment as shown in Figure5, which needs the following hardware: solar panels, switches $T_{c}$, diodes VDC, capacitors $C_{s}$, capacitors $C_{d}$, and inductors $L_{c}$. The simulation process requires analog solar power, DSP emulators, DSP circuit boards, etc. The various hardware parameters are shown as follows.

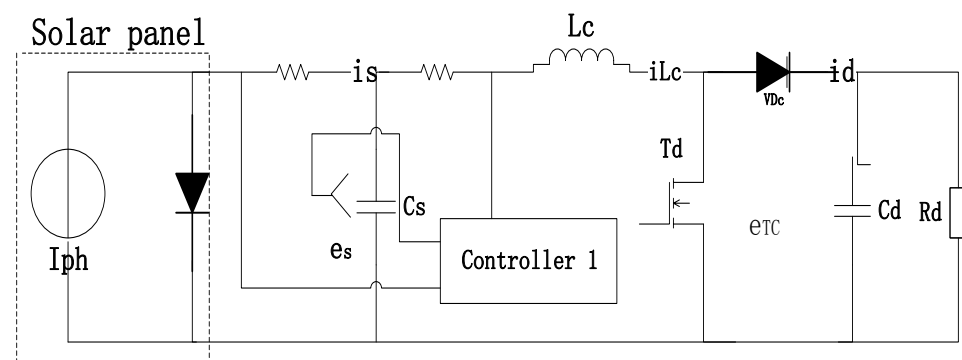

Figure 5. DC/DC Boost Circuit

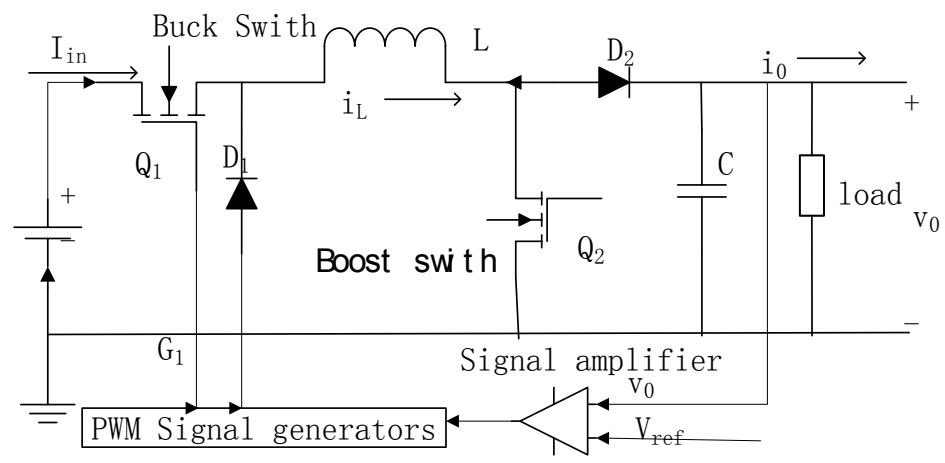

Figure 6. The Block Diagram Of DC/DC Converter System

When the outside temperature is kept constant, the solar maximum power point remained around a voltage according to the characteristics of solar modules curve. The experimental demonstrate the ratio of the maximum power point voltage and the open circuit voltage is a constant 0.75 . When the output voltage of the solar module is 0.75 
times of the open circuit voltage, reach to the maximum output power of solar modules. Therefore, we will access DC/DC converter to solar modules input circuit, switch duty cycle controlled by the microcontroller to control the output voltage of the solar module to reach the maximum power point voltage to achieve the solar maximum power point tracking. The method uses simple circuit, hardware and software, and the control method is flexible. The mode equivalent circuit of each switching cycle is shown in Figure 7. Buck and boost mode does not appear in the same switching cycle.

In buck mode, boost switch $\mathrm{Q}_{2}$ is off. When the buck switch $\mathrm{Q}_{1}$ closed, the converter turns into working mode, at this point the inductor current has a minimum, and the diode withstand negative voltage forcing its shutdown, so the current makes the inductor current increases linearly through the inductor, and inductive charging in this sub-section; when the control circuit commands the buck switch $\mathrm{Q}^{1}$ is off, the inductor current will force the diode to conduct, the inductor current decreases. The working mode end since the switch is closed again, the inductor current increases again and takes one time cycle.

In boost mode, the buck switch $\mathrm{Q}_{1}$ is off. Assuming before closing and opening, a small current flowing through the inductor when the boost switch $\mathrm{Q}_{2}$ is closed, the inductor current is linearly increased, the reverse voltage forced diode to be shutdown. Therefore, no current flows through the diode and the load is fully powered by the output capacitor. Inductor current linearly increases will continue until the boost switch $\mathrm{Q}_{2}$ is off. When $\mathrm{Q}_{2}$ turns off, the energy stored in the inductor begins to consume, so that the diode is conducting, one part the inductor provide energy to the load, the other for charging the capacitor. When the inductor current continues to decrease until the boost switch $\mathrm{Q}_{2}$ is closed again or current approaches 0 , the switch is closed again before the inductor current is 0 , so that the current gradually increases and to reach the cycle.

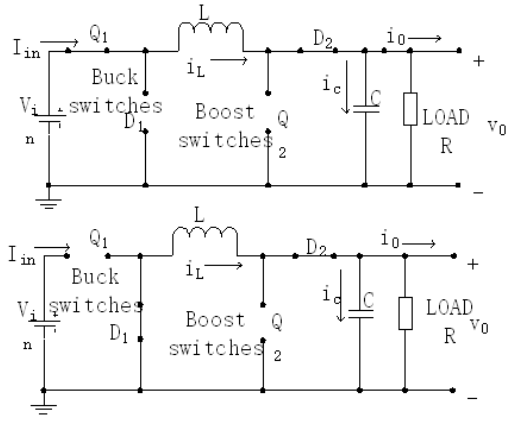

a) Buck mode

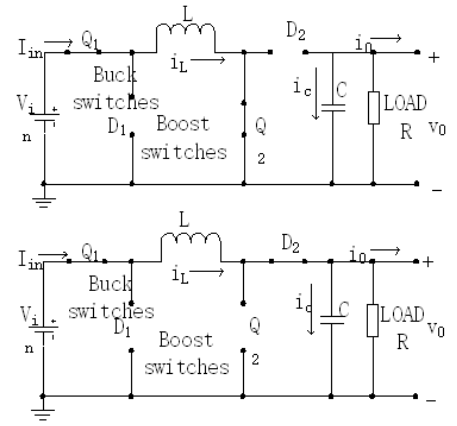

b) Boost mode

Figure 7. The Equivalent Circuit of DC/DC Converter Mode

Since the buck-boost mode does not occur simultaneously in the same control period, use the state-space averaging technique on modeling the DC/DC converter. For small signal modeling:

$$
\begin{gathered}
i_{L}=I_{L}+\tilde{i}_{L} v_{0}=V_{0}+\tilde{v}_{0} v_{\text {in }}=V_{\text {in }}+\tilde{v}_{\text {in }} \tilde{v}_{\text {in }} \approx 0 \\
d_{\text {buck }}=D_{\text {buck }}+\tilde{d}_{\text {buck }}=d_{c t r l} \leq 1 d_{\text {boost }}=D_{\text {boost }}+\tilde{d}_{\text {boost }}=d_{c t r l}-1 \geq 0 \\
d_{\text {buckboost }}=D_{\text {buckboost }}+\tilde{d}_{\text {buckboost }}
\end{gathered}
$$

In buck mode, the DC gain and the transfer function is given as:

$$
\begin{gathered}
\frac{d_{i_{L}}}{d t}=\frac{1}{L}\left(d_{\text {buck }} v_{\text {in }}-v_{0}\right) \\
\frac{d_{v_{0}}}{d t}=\frac{1}{C}\left(i_{L}-\frac{v_{0}}{R}\right)
\end{gathered}
$$


In boost mode, the DC gain and the transfer function:

$$
\begin{aligned}
& \frac{d i_{L}}{d t}=\frac{1}{L}\left[v_{\text {in }}-\left(1-d_{\text {boost }}\right) v_{0}\right] \\
& \frac{d v_{0}}{d t}=\frac{1}{C}\left[\left(1-d_{\text {boost }}\right) i_{L}-\frac{v_{0}}{R}\right]
\end{aligned}
$$

As can be seen from the above formula, DC/DC converter output voltage is adjusted by the parameters, in buck mode, when the duty ratio is close to 1 , the inductor current will become very large, the interference is at the output voltage according to the formula (20). Similarly, in boost mode, when the duty ratio is very low, the inductor current as shown in equation (22) will be the interference of the output voltage.

\subsection{Software Implementation}

The control unit is the key part of the whole solar power generation system, comprehensive management of the whole system. According to the environmental conditions, the controller should be able to make full use of solar energy and improve energy efficiency, set the key parameters according to the characteristics of storage battery, such as the battery overcharge and over discharge point. Restoring the connection point, it should also have the light control and time control function when the load is light.

The solar power generation system control architecture is shown in Figure8. First, it controls the output voltage of the solar module around the maximum power point voltage by DC/DC converter to achieve maximum power output of solar energy. Secondly, supplying the power to the battery or load, then control the battery by the battery charging and discharging circuit.

We need ensure the battery overcharge and over discharge.

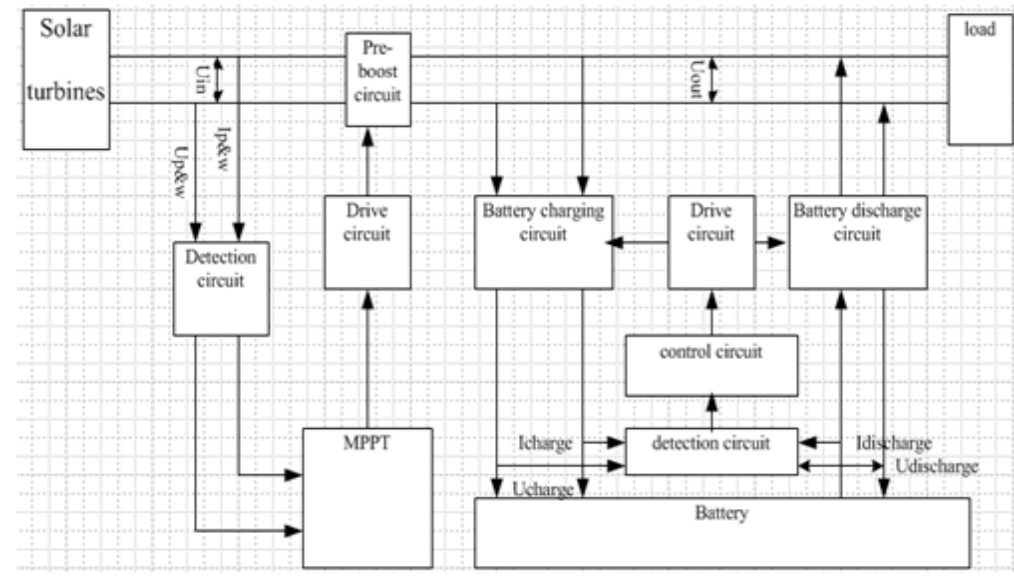

Figure 8. The Control Architecture of Solar Power Generation System

There is exponential function computing in the formula, so we choose the PC56F8245 chip to program, which can ensure the computing speed.

\subsection{Experimental procedure and results}

In the concrete experiment, the inverter and paralleling in the grid process are not be considered, so the process of connecting a resistance in boosted DC/DC circuit is shown in Figure 9. However, the sunshine and temperature changes a bit in a short time, so the period for sampling should set to 10 minutes due to the cycle of maximum power point range between 0 and 0.5 . When setting the starting value of duty cycle to 0.5 , it decreases gradually by 0.01 each time, and then do recursive computation. In the experiment, the 
minimum value improve up to 0.1 , the maximum value improve up to $28.5 \%$ as shown in Figure 9.

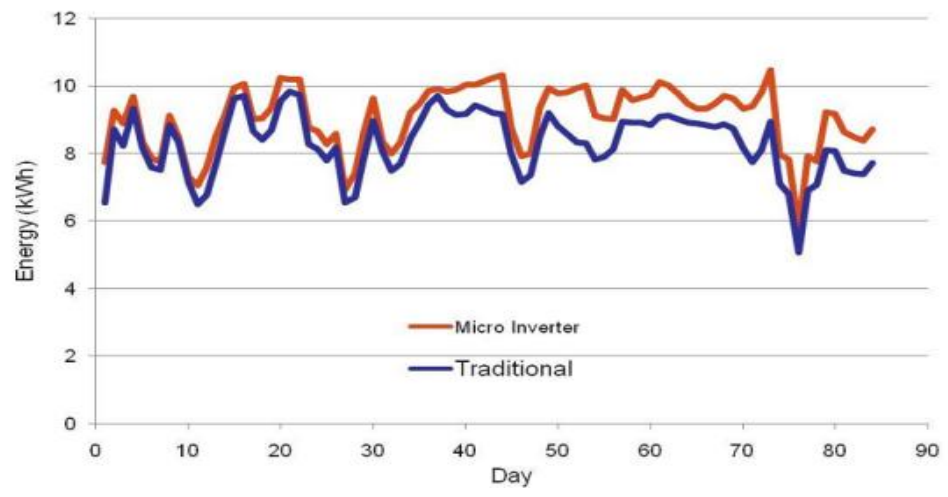

Figure 9. The Comparison of Experiment Results

There are many advantages as follows. The PV modules do not need to match, which can be consisted of any combination of any firm products with different specifications and different life situations; without limited to the laying of a unified direction and different orientations of the entire roof can be covered. And this micro-inverters can contribute more than $16 \%$ compared to conventional inverters, and make full use of fragmented landform, occasionally even the shade area, so as a conservative estimate under the same generation, the inverse system can save 19-26\% of the occupied land area. It has low voltage starting and good weak light characteristics, and high system efficiency and $85-90 \%$ guarantee value, the efficiency is the basic guarantee for the more electricity. Efficiency of each 5\% improvement is equal to the component $1 \%$ efficiency; maintenance phase tracking location and fast repair, and no system outage.

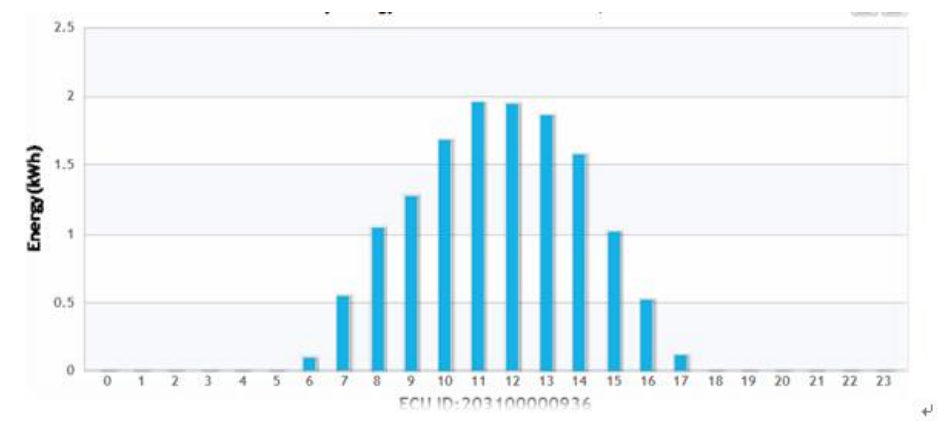

Figure 10. The Generation Effect of The Actual Measurement

\section{Conclusion}

The principle of distributed least action MPPT control method is proposed to overcome the difference between the electrical parameters of the solar cell and the actual difference in solar radiation. The energy loss caused by different solar module strings in the different direction is compared to the general application of the MPPT, which improves the power generation efficiency of the system. The proposed least action recursive method can be accurate when tracking the solar panels to the maximum power point when the sunlight intensity, ambient temperature and load resistance change a lot. The system has good stability and reduces the loss of the circuit. For effective use of solar energy, the maximum power point tracking technology will be widely used in photovoltaic power generation system which can achieve greater practical value and social benefits. 


\section{References}

[1] W. Zhao, "Research on Grid Connected Photovoltaic System", Hefei University of Technology, (2003).

[2] W. T. Li, H. Liu, H. L. Chen and J. Qinghai, "Electric Power", vol. 23, no. 3, (2005).

[3] Y. L. Shen, J. H. Su, W. Zhao and J. Acta, "Energiae Solaris Sinic", vol. 24, (2003), pp. 655.

[4] N. Femia and G. J. Petrone, "IEEE Transactions on power electronics", vol. 20, (2005), pp. 963.

[5] N. S. D’Souza, L. C. Lopes and X. J. Liu, “An Intelligent Maximum Power Point Tracker using Peak Current Control”, IEEE 36th Conference on Power Electronics Specialists, Recife, Brazil, IEEE, (2005), pp. 172-177.

[6] Y. T. Hsiao and C. H. Chen, "IEEE PESC", vol. 12, (2009), pp. 37.

[7] M. Verrachary, K. Uezato and K. Uezato, "IEEE Transactions on Aerospace and Electronic System", vol. 38, (2002), pp. 969.

[8] K. H. Chao, C. J. Li and M. H. Wang, "LNCS”, vol. 5551, (2009), pp. 745.

[9] N. Femia, G. Petrone and C. Spagnuolo, "Optimizing, Duty-cycle Perturbation of P\&O, MPPT technique", IEEE 35th Annual Power Electronics Specialists Conference. Aachen, Germany, IEEE, (2004), pp. 1939-1944.

[10] B. Y. Liu, X. S. Duan, F. Liu and P. W. Xu, "Transactions of China Electro technical Society", vol. 24, (2009), pp. 91.

[11] F. R. Liu and H. X. Duan, "IEEE Transactions on Industrial Electronics", vol. 55, (2008), pp. 2622.

[12] F. Luo and P. W. Xu, "IEEE Conference on Industrial Electronics and Applications", no. 2, (2007), pp. 2259.

[13] S. J. Chiang, K. T. Chang and C. Y. Yen, "IEEE Trans, Ind. Electron”, vol. 45, (1998), pp. 38.

[14] C. Hua and C. Shen, "IEEE PESC", vol. 1, (1998), pp. 86.

[15] Y. C. Kuo, T. J. Liang and J. F. Chen, "IEEE Trans. Ind. Electron”, vol. 48, (2001).

[16] Y. P, M. M and Jovanovich, "IEEE Trans, Power Electron", vol. 17, (2002).

[17] H. Wang, C. Gong, H. Ma and Y. Yan, "IEEE ICIEA'06", (2006).

[18] F. Forest, E. Lamoure, T. A. Maynard and J. J. Huselstein, "IEEE Trans Power Electron", vol. 22, (2007), pp. 1662.

[19] T. Qian and B. Lehman, "IEEE Trans, Power Electron", vol. 23, (2008), pp. 88.

[20] G. C. Hsieh, H. L. Chen, Y. Chen, C. M. Tsai and S. S. Shyu, "IEEE APEC", (2008).

\section{Authors}
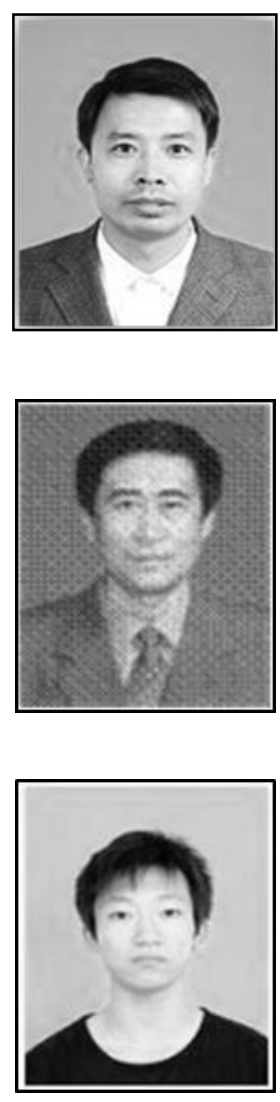

Xiaoju Yin, received the MBA degree from Liaoning University, Shenyang, China in 2007. He is currently an associate professor with the School of Renewable Energy, Shenyang Institute of Engineering, and working towards the Ph.D degree in Electric Engineering in Shenyang University of Technology. His current research interests include the energy storage technology and intelligent control theory.

Fengge Zhang, received the Ph.D degree in Electrical Engineering from Shenyang University of Technology, Shenyang, China, in 2000. $\mathrm{He}$ is currently a professor with the College of Electrical Engineering, Shenyang University of Technology. His research interests include electromagnetic theory, optimized design for power converter etc.

Yonggang Jiao, received the B.E. degree in Electrical Engineering and Automation from Shenyang University of Technology, Shenyang, China in 2012. He is currently working towards the Master degree in Electric Engineering in Shenyang University of Technology. 


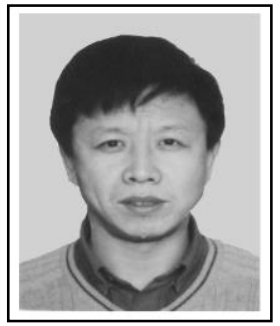

Zhenhe Ju, received the Master degree in Solar Engineering from Europe Solar Research Centre, Stockholm, Sweden, in 2004. He is currently a professor with the School of Renewable Energy, Shenyang Institute of Engineering, Shenyang. His current research interests include solar photovoltaic application technology and green lighting technology, etc. 
International Journal of Grid Distribution Computing Vol. 8, No.4, (2015) 